\title{
Associação entre padrão alimentar e perfil lipídico em adolescentes de escolas públicas em Salvador, Bahia.
}

Association between dietary pattern and lipid profile in adolescents from public schools in Salvador, Bahia.

Asociación entre patrón dietético y perfil lipídico en adolescentes de escuelas públicas en Salvador, Bahía.

\author{
Josiene Carvalho Pereira \\ ORCID: https://orcid.org/0000-0002-5557-578X \\ Universidade Federal da Bahia, Brasil \\ E-mail: josienecp@yahoo.com.br \\ Maria Ester Pereira da Conceição-Machado \\ ORCID: https://orcid.org/0000-0001-5982-4213 \\ Universidade Federal da Bahia, Brasil \\ E-mail: marester@ufba.br \\ Mônica Leila Portela Santana \\ ORCID: https://orcid.org/0000-0002-2706-8238 \\ Universidade Federal da Bahia, Brasil \\ E-mail: monicalportela@gmail.com \\ Rita de Cássia Ribeiro-Silva \\ ORCID: https://orcid.org/0000-0002-8387-9254 \\ Universidade Federal da Bahia, Brasil \\ E-mail: rcrsilva@ufba.br \\ Priscila Ribas de Faria Costa \\ ORCID: https://orcid.org/0000-0003-3809-9037 \\ Universidade Federal da Bahia, Brasil \\ E-mail: priscilarf@yahoo.com.br \\ Elizabete Jesus Pinto \\ ORCID: https://orcid.org/0000-0003-0335-8705 \\ Universidade Federal da Bahia, Brasil \\ E-mail: elizabetepinto@yahoo.com.br \\ Ricardo David Couto \\ ORCID: https://orcid.org/0000-0003-2119-437X \\ Universidade Federal da Bahia, Brasil \\ E-mail: rdc@ufba.br \\ Ana Marlúcia Oliveira \\ ORCID: https://orcid.org/0000-0002-8162-4274 \\ Universidade Federal da Bahia, Brasil \\ E-mail: amoa@ufba.br
}

\begin{abstract}
Resumo
Este estudo investigou a relação entre padrões alimentares e perfil lipídico em adolescentes. Trata-se de estudo transversal envolvendo amostra probabilística de 1.188 estudantes de 11 a 18 anos, de escolas públicas da cidade de Salvador-Bahia. O consumo alimentar foi analisado através do questionário de frequência alimentar semi-quantitativo e os padrões alimentares foram identificados utilizando da análise fatorial pela técnica de componentes principais e os escores categorizados em tercis. Associações entre padrão alimentar com o perfil lipídico (colesterol total, colesterol da lipoproteína de alta densidade, colesterol da lipoproteína de baixa densidade e triglicerídeos) foram realizadas por regressão logística politômica ajustadas por sexo, idade e estado nutricional. Identificou-se três padrões alimentares caracterizados em: não saudável, saudável e tradicional. Nos modelos ajustados, foi encontrada associação significante entre consumo alimentar no menor tercil do padrão saudável, com concentração alta de triglicerídeos (odds ratio $=1,88$; intervalo de confiança $95 \%=1,10-3,22$ ) e baixas de colesterol da lipoproteína de alta densidade (odds ratio: 1,62; intervalo de confiança 95\% =1,01-2,62), quando comparados ao consumo no maior tercil desse padrão. Foi identificada associação entre menor aderência ao padrão saudável com níveis séricos baixos de lipoproteína de alta densidade e altos de triglicerídeos. Não houve associação entre os padrões não saudável e tradicional com o perfil lipídico.
\end{abstract}

Palavras-chave: Adolescente; Consumo alimentar; Perfil lipídico; Padrão alimentar. 


\begin{abstract}
This study investigated the relationship between dietary patterns and lipid profile in adolescents. This is a crosssectional study involving a probabilistic sample of 1,188 students aged 11 to 18 years, from public schools in the city of Salvador-Bahia. Food consumption was analyzed using a semi-quantitative food frequency questionnaire and dietary patterns were identified using factor analysis using the principal component technique and scores categorized into tertiles. Associations between dietary pattern and lipid profile (total cholesterol, high-density lipoprotein cholesterol, low-density lipoprotein cholesterol, and triglycerides) were performed by polytomous logistic regression adjusted for sex, age, and nutritional status. Three dietary patterns were identified, characterized as: unhealthy, healthy and traditional. In the adjusted models, a significant association was found between food consumption in the lowest tertile of the healthy pattern, with high concentration of triglycerides (odds ratio $=1.88$; 95\% confidence interval $=1.10-3.22$ ) and low cholesterol levels high-density lipoprotein (odds ratio: 1.62; $95 \%$ confidence interval = 1.01-2.62), when compared to consumption in the highest tertile of this pattern. An association was identified between lower adherence to the healthy pattern with low serum levels of high-density lipoprotein and high triglycerides. There was no association between unhealthy and traditional patterns and lipid profile.
\end{abstract}

Keywords: Adolescent; Food consumption; Lipid profile; Dietary pattern.

\title{
Resumen
}

Este estudio investigó la relación entre los patrones dietéticos y el perfil lipídico en adolescentes. Se trata de un estudio transversal que involucra una muestra probabilística de 1.188 estudiantes de 11 a 18 años de escuelas públicas de la ciudad de Salvador-Bahía. El consumo de alimentos se analizó mediante un cuestionario semicuantitativo de frecuencia de alimentos y los patrones dietéticos se identificaron mediante el análisis factorial utilizando la técnica del componente principal y las puntuaciones categorizadas en terciles. Las asociaciones entre patrón dietético y perfil lipídico (colesterol total, colesterol unido a lipoproteínas de alta densidad, colesterol unido a lipoproteínas de baja densidad y triglicéridos) se realizaron mediante regresión logística politómica ajustada por sexo, edad y estado nutricional. Se identificaron tres patrones dietéticos, caracterizados como: insalubre, saludable y tradicional. En los modelos ajustados, se encontró asociación significativa entre el consumo de alimentos en el tercil más bajo del patrón saludable, con alta concentración de triglicéridos (odds ratio = 1,88; intervalo de confianza del 95\% = 1,10-3,22) y niveles bajos de colesterol en los niveles altos de colesterol. lipoproteínas de densidad media (odds ratio: 1,62; intervalo de confianza del $95 \%=1,01-2,62$ ), en comparación con el consumo en el tercil más alto de este patrón. Se identificó una asociación entre una menor adherencia al patrón saludable con niveles séricos bajos de lipoproteínas de alta densidad y triglicéridos altos. No hubo asociación entre los patrones tradicionales y no saludables y el perfil lipídico.

Palabras clave: Adolescente; Consumo de alimentos; Perfil lipídico; Patrón dietético.

\section{Introdução}

A adolescência é um ciclo da vida no qual ocorrem intensas mudanças corporais, físicas e comportamentais, repercutindo nas escolhas, em especial na construção dos hábitos alimentares (Corrêa, Vencato, Rockett \& Bosa, 2017). Quando as escolhas alimentares são inadequadas podem comprometer o estado nutricional e de saúde, favorecendo o aparecimento de Doenças Crônicas Não Transmissíveis (DCNT) ao longo da vida (Corrêa et al., 2017; Berenson, Srinivasan, Xu \& Chen, 2016).

Anormalidades lipídicas entre adolescentes integram as evidências científicas, construídas a partir de estudos nacionais e internacionais, indicando a necessidade de rastreamento contínuo do perfil lipídico no intuito de fornecer o diagnóstico precoce de alterações lipídicas associadas ao desencadeamento do processo aterosclerótico e de gerar informações para que intervenções de saúde pública sejam realizadas prevenindo o desenvolvimento de eventos cardiovasculares na vida adulta (Berenson et al, 2016; White et al., 2019; Enes \& Silva, 2018; Lartey, Marquis, Aryeetey \& Nti, 2018). No entanto, a avaliação do perfil lipídico na adolescência deve ser realizada de forma criteriosa, pois as concentrações de lipoproteínas mudam expressivamente com o crescimento e maturação sexual, tornando ainda mais específica essa fase da vida (Jolliffe \& Janssen, 2006).

O padrão alimentar pouco saudável, caracterizado principalmente pelo consumo de produtos industrializados, prontos para consumo e de baixa qualidade nutricional, pode influenciar de forma desfavorável o crescimento somático, além de contribuir para predisposição a DCNT, como alterações cardiometabólicas, dentre elas as anormalidades do perfil lipídico (Carvalho et al., 2016). Embora a produção do conhecimento da relação entre consumo alimentar e marcadores de risco 
cardiovascular seja consistente para adultos e, ainda que a saúde cardiometabólica seja fortemente dependente do estilo de vida, para os adolescentes ainda são necessárias maiores investigações. Apesar da existência de estudos internacionais nesta temática (Carvalho et al., 2016; Zhang et al., 2005; Mirmiran et al., 2019; Perng et al., 2017), no Brasil ainda são poucos os estudos que abordam a relação entre perfil lipídico e padrão de consumo alimentar entre adolescentes (Dishchekenian et al., 2011; de Alcântara Neto, Silva, Assis \& Pinto, 2012; Vaz et al., 2018).

Destacando a importância da produção de evidências consistentes sobre o tema para embasar políticas públicas relacionadas à prevenção de doenças cardiometabólicas na vida adulta e o desenvolvimento dos fatores de risco na adolescência, este estudo teve objetivo de investigar a relação entre padrões alimentares e perfil lipídico em adolescentes.

\section{Metodologia}

Estudo transversal, inserido em uma investigação mais ampla, intitulada "Fatores psicossociais como elementos que repercutem nas condições de saúde, nutrição e desenvolvimento cognitivo de estudantes do ensino fundamental de escolas públicas de Salvador/BA”, envolvendo amostra probabilística de estudantes de 11 a 18 anos, de ambos os sexos. O protocolo de estudo foi aprovado pelo Comitê de Ética em Pesquisa do Instituto de Saúde Coletiva, parecer 002-08 CEP/ISC, registro 051-07/CEP-ISC e da Escola de Nutrição da Universidade Federal da Bahia (parecer 14/12). Todos os participantes tiveram autorização escrita por meio do Termo de Consentimento Livre e Esclarecido (TCLE), assinada pelos pais ou responsáveis legais.

Para definição da amostra foram obtidas informações do censo escolar 2007, disponibilizadas pela Secretaria de Educação do Estado da Bahia. A amostra probabilística baseou-se na população de 77.873 estudantes matriculados nas escolas estaduais de Salvador, adotando-se a técnica de conglomerados em dois estágios (escolas e turmas). Foram selecionadas aleatoriamente 23 das 207 escolas estaduais e os estudantes foram captados a partir do sorteio de três turmas por escola e avaliados todos os alunos dessas turmas que concordaram em participar. Adolescentes gestantes, nutrizes e com problemas físicos que impossibilitassem avaliação antropométrica não foram incluídos do estudo. Considerando que esta amostra não foi estimada para atender ao objetivo desta investigação, foi calculado o erro amostral a posteriori adotando-se prevalência de 7,2\% para concentrações altas de triglicerídeos (TG), 5,9\% para Colesterol Total (CT), 5,8\% para colesterol da Lipoproteína de Baixa Densidade (LDL-c) e 25,7\% para baixa concentração de colesterol da Lipoproteína de Alta Densidade (HDL-c), obtendo o número amostral mínimo de 1.045 adolescentes para avaliar todas as associações de interesse, com um nível de confiança de 95\% e margem de erro máximo admissível de 3\%. A amostra deste estudo foi constituída por 1.212 adolescentes, sendo excluídos 24 devido ausência de dados antropométricos e dietéticos, resultando em 1.188 participantes, número maior que o cálculo amostral mínimo necessário para avaliar as associações de interesse.

A coleta de dados foi realizada no ambiente escolar por equipe qualificada e treinada composta por nutricionistas, estudantes de nutrição e técnico de enfermagem, no período de julho a dezembro de 2009. Foram coletados $10 \mathrm{~mL}$ de sangue por punção venosa em tubos vacutainer $\left(\mathrm{BD}^{\circledR}\right)$ estéreis e descartáveis. As concentrações séricas de CT, HDL-c e TG foram determinadas pelo método enzimático. O LDL-c foi calculado considerando a equação de Friedewald (LDL-c = CT - HDL-c TG/5), quando os valores de TG estiveram abaixo de $400 \mathrm{mg} / \mathrm{dL}$ (Faludi et al., 2017).

Os pontos de corte para avaliação do perfil lipídico foram os preconizados por Jolliffe e Janssen (2006), para escolares de 12 a 18 anos. Para os escolares menores de 12 anos, adotou-se a recomendação do National Health and Nutrition Examination Survey (NHANES) para classificar o HDL-c e do National Cholesterol Education Program (NCEP) para classificação do CT, LDL-c e TG (Magnussen et al., 2008).

O peso e altura foram aferidos em duplicata, por dois avaliadores independentes, adotando a média entre os valores medidos. O peso foi obtido por meio da balança digital portátil Master ${ }^{\circledR}$ (Brasil) e altura aferida pelo estadiômetro portátil da 
marca Leicester Height Measure ${ }^{\circledR}$ (Hamburgo, Alemanha) graduado em decímetros de centímetros. Foram desprezadas medidas com diferença maiores de $100 \mathrm{~g}$ para o peso, assim como variações maiores que $0,1 \mathrm{~cm}$ na aferição da altura (Lohman, Roche \& Martorell, 1988).

O estado nutricional foi avaliado pelo Índice de Massa Corporal por idade (IMC/I), preconizado pela Organização Mundial da Saúde (OMS), conforme o sexo, considerando: magreza (< percentil 3), adequado ( $\geq$ percentil 3 e $\leq$ percentil 85 ), sobrepeso (> percentil 85 e $\leq$ percentil 97) e obesidade (percentil > 97) (World Health Organization [WHO], 2007). Para análise foi considerado com excesso de peso (IMC/I > percentil 85) e sem excesso de peso (IMC/I < percentil 85).

Para avaliar o estrato econômico foi utilizado o Critério de Classificação Econômica Brasil (CCEB), que considera a posse de bens domésticos e a escolaridade do chefe da família, classificando em categorias econômicas que variam de A (melhor estrato) a E (pior estrato) [(Associação Brasileira de Empresas de Pesquisa (ABEP), 2008]. O consumo alimentar foi avaliado por meio do Questionário de Frequência Alimentar (QFA) Semi-quantitativo, considerando o consumo nos últimos seis meses.

O QFA constituído por 97 itens alimentares com cinco possibilidades de respostas: nunca/raro, uma a três vezes ao mês, uma vez por semana, duas a quatro vezes na semana ou mais de quatro vezes na semana, validado pelo grupo de pesquisa (Mascarenhas et al., 2016). O QFA foi auto aplicado aos adolescentes e posteriormente 10\% do total de todos os questionários respondidos foram reaplicados pelos pesquisadores para checar a qualidade dos dados obtidos do auto relato. Os itens alimentares do QFA foram combinados em 19 grupos com base na composição de nutrientes e consumo (Quadro 1).

A análise fatorial utilizando a técnica de Componentes Principais foi utilizada para reduzir os 19 grupos alimentares fatores (padrões) para explicar as variações na ingestão alimentar. As frequências de consumo de cada alimento foram convertidas em frações diárias (escores), multiplicando a frequência semanal por 4 (número de semanas no mês) e dividindo-a por 30 (número de dias no mês), encontrando-se assim, o escore de consumo médio diário para cada alimento (Neumann, Martins, Marcopito \& Araujo, 2007), utilizando apenas uma unidade de análise.

Para o processamento e construção do banco de dados foi utilizado o programa Epi-Info versão 6.0, adotando a digitação dupla dos dados. Realizou-se a análise descritiva, utilizando medidas de tendência central, frequência e prevalência. Foi avaliada a adequação da amostra para a análise fatorial e a mesma atendeu tanto o critério de ter no mínimo dez participantes para cada item alimentar contido no QFA, quanto ao número de participantes ter sido no mínimo cinco vezes maior do que o número de itens do QFA, necessários para este tipo de análise (Hair et al., 2009).

Quadro 1. Descrição dos grupos de alimentos utilizados na análise do padrão alimentar em adolescentes de escolas públicas de Salvador, Bahia, Brasil, 2009.

\begin{tabular}{|c|c|}
\hline Grupos Alimentares & Itens alimentares \\
\hline Açúcares e doces & $\begin{array}{l}\text { Açúcar de adição nos líquidos, balas/chicletes/pirulitos, chocolate em barra, doces industrializados (goiabada e } \\
\text { marmelada), doces caseiros, sorvete/picolé de creme/chocolate, cremosinho, açaí, farinha láctea/Neston, } \\
\text { achocolatado em pó, gelatina e granola. }\end{array}$ \\
\hline Bebidas artificiais & $\begin{array}{l}\text { Refrigerante tradicional, refrigerante diet/light, refresco artificial, bebida gaseificada, raspadinha/geladinho } \\
\text { artificial, suco artificial e bebida energética. }\end{array}$ \\
\hline Bolos e Biscoitos & Bolo caseiro, biscoito salgado/doce, bolo industrializado e biscoito recheado. \\
\hline Café e Chá & Café e Chá. \\
\hline $\begin{array}{l}\text { Carne bovina } \\
\text { e vísceras }\end{array}$ & Carne bovina frita ou cozida, vísceras (fígado, rins) e carne de sertão/charque/de sol fritas. \\
\hline Cereais e farinhas & $\begin{array}{l}\text { Arroz branco ou integral, macarrão branco ou integral, farináceos (aveia, gérmen de trigo), milho/cuscuz de } \\
\text { milho e farinha de mandioca. }\end{array}$ \\
\hline Comidas típicas & Acarajé/abará/vatapá/caruru, feijão tropeiro, feijoada/dobradinha e marisco. \\
\hline Feijão & Feijão preto, verde, mulatinho, fradinho, ranço, andu, ervilha, grão de bico, fava, mangalô. \\
\hline Frango e Ovos & Frango com ou sem pele (frito ou cozido) e ovo cozido/frito/preparações com ovos. \\
\hline
\end{tabular}




\begin{tabular}{|l|l|}
\hline Frutas & $\begin{array}{l}\text { Suco de fruta ou polpa de fruta/geladinho, abacaxi, abacate, acerola, banana da prata, banana da terra, caju, } \\
\text { cajá, jaca, mamão, manga, maçã, melancia, melão, laranja, tangerina e morango. }\end{array}$ \\
\hline Lanches & $\begin{array}{l}\text { Embutidos (calabresa, presunto, mortadela e salsicha), pizza/lasanha, sopa industrializada, macarrão } \\
\text { instantâneo, preparações fritas (pastel, coxinha, sonho, quibe e churros), cachorro quente, sanduíche, batata } \\
\text { frita ou palha, salgadinho (milho e queijo) e pipoca doce/salgada. }\end{array}$ \\
\hline Leite & Leite integral em pó ou líquido e leite desnatado em pó ou líquido. \\
\hline Molhos & Margarina, molho pronto para salada, molho pronto para pizza, lasanhas, ketchup e maionese. \\
\hline Óleos e gorduras & Óleo, azeite de oliva, manteiga, azeite de dendê, leite de coco, amendoim, castanhas e nozes. \\
\hline Pão & Pão branco ou integral. \\
\hline Peixes & Peixe cozido ou frito. \\
\hline Produtos lácteos & $\begin{array}{l}\text { Queijo amarelo, queijo branco, requeijão cremoso integral/ "light", iogurte integral/ "diet/light", achocolatado } \\
\text { pronto e leite fermentado. }\end{array}$ \\
\hline Raízes e tubérculos & Batata doce, aipim, inhame, cará, inhaminho. \\
\hline Verduras e Legumes & $\begin{array}{l}\text { Alface, couve, abóbora, batata inglesa, beterraba, cenoura, chuchu, maxixe, quiabo, sopa de legumes com } \\
\text { massa, sopa de legume, repolho e tomate. }\end{array}$ \\
\hline
\end{tabular}

Fonte: Autores.

Para verificar a adequação dos dados ao método de análise fatorial foram aplicados dois testes estatísticos: KaiserMeyer-Olkin (KMO) e o Teste de Esfericidade de Bartlett (BTS). O KMO verifica a adequação da amostra à técnica de análise fatorial, o quociente resultante pode variar entre 0 e 1 e resultados menores do que 0,6 indicam que a técnica não se aplica a base de dados para a análise fatorial (Hair et al., 2009). O BTS indica se o modelo fatorial é adequado para a análise dos dados quando a significância estatística for igual ou menor a 0,05. Posteriormente foi realizada a rotação dos fatores por meio da rotação ortogonal Varimax para melhor interpretabilidade dos dados (Hair et al., 2009; Borges et al., 2015).

Os critérios utilizados para definir o número de fatores a serem retidos na análise fatorial foram: autovalor > 1, variância total, Cattel test graph (scree plot), comunalidade (valor mínimo de 0,20) e interpretabilidade das cargas fatoriais (Neumann et al., 2007; Hair, 2009; Borges et al., 2015; Ribeiro-Silva et al., 2018). Os fatores foram selecionados, interpretados como padrões alimentares e nomeados em Padrão Saudável, Padrão Não Saudável e Padrão Tradicional, de acordo com as características dos itens alimentares retidos, utilizando-se nomenclatura semelhante à de outros estudos com a mesma metodologia (Dishchekenian et al., 2011; Vaz et al., 2018; Mascarenhas et al., 2016; Borges et al., 2015; Ribeiro-Silva et al., 2018).

Grupos alimentares com cargas fatoriais > 0,25 foram considerados importantes componentes do padrão alimentar. Cargas fatoriais positivas $(>0,25)$ indicaram correlações positivas entre o grupo alimentar e os fatores; cargas fatoriais negativas $(<-0,25)$ apresentaram correlações negativas entre o grupo alimentar e o fator (Vaz et al., 2018; Borges et al., 2015). Realizou-se o cálculo dos escores fatoriais para cada participante do estudo (Neumann et al., 2007; Borges et al., 2015). Os escores foram estratificados em tercis e constituíram as variáveis independentes principais. Variáveis lipídicas foram categorizadas em normal (referência), limítrofe e alto (CT, LDL-c, TG) e baixo (HDL-c). O padrão alimentar foi categorizado em tercil 1, tercil 2 e tercil 3 (referência), caracterizando em ordem crescente a frequência de consumo.

As covariáveis foram representadas pela idade ( $<14$ anos e $\geq 14$ anos), sexo (feminino e masculino), categoria econômica (classe C/D e classe E) e estado nutricional, caracterizado "sem excesso de peso (magreza/eutrofia)" e "com excesso de peso (sobrepeso/obesidade) ". Foi realizado um modelo de regressão logística politômica, utilizando como estimador a Odds Ratio (OR) e o respectivo Intervalo de Confiança (IC95\%), para cada variável desfecho em função das variáveis de exposição principal. Optou-se por utilizar a regressão logística politômica por permitir a categorização da variável resposta (perfil lipídico) em um número maior de categorias. Inicialmente, realizou-se a análise simples e selecionou as variáveis que apresentaram $\mathrm{p}$ valor $<0,20$ para o modelo múltiplo permanecendo no modelo final aquelas com $\mathrm{p}$ valor $<0,05$. 
Possíveis fatores de interação foram testados para avaliar a presença de modificação de efeito entre as variáveis de exposição e as variáveis desfecho, utilizando o teste da razão de log-verossimilhança. A presença de variáveis de confusão (associadas tanto com a exposição quanto com o desfecho) foi testada e a variação maior que 10\% na OR múltiplas comparada com a OR do modelo simples foi considerada confundimento (Greenland, 2008).

O Akaike Information Criterion (AIC) foi usado para avaliar a qualidade do ajuste do modelo estatístico baseado na medida relativa de perda da informação ao adotar determinado modelo (Akaike, 1973). As análises dos dados foram realizadas pelo pacote estatístico Stata 12.0. A correção do delineamento complexo da amostra ocorreu com o comando SVY.

\section{Resultados}

A maioria $(59,7 \%)$ dos adolescentes era do sexo feminino; 54,5\% com idade maior ou igual a 14 anos, média de idade igual 14,29, Desvio Padrão (DP) = 1,53 e 49,8\% pertencentes à categoria econômica E. Em relação ao estado nutricional, $15,2 \%$ dos adolescentes estavam com excesso de peso (9,3\% com sobrepeso e 5,9\% obesos). Observou-se que 25,7\% dos adolescentes tinham HDL-c baixo, seguido de alterações no CT (5,9\%), LDL-c (5,8\%) e TG (7,2\%) (Tabela 1). Tanto os resultados do KMO $(0,938)$ quanto o BTS $\left(\chi^{2}=9630,544 ; \mathrm{p}<0,001\right)$ indicaram que os dados da amostra estão adequados à aplicabilidade do modelo fatorial e a confiabilidade dos resultados encontrados. A partir da análise fatorial, foram identificados três padrões alimentares da população em estudo, explicando 53,98\% da variabilidade total dos dados (Tabela 2).

Os resultados das análises da regressão logística politômica estão apresentados na Tabela 3. A análise simples indicou que a aderência ao menor tercil do Padrão Saudável foi associada as altas concentrações de TG (OR=1,80; IC95\%: 1,06-3,07, $\mathrm{p}=0,028)$ e baixas de HDL-c (OR=1,48; IC95\%: 0,93-2,37, $\mathrm{p}=0,095)$, quando comparados aos adolescentes que aderiram ao maior tercil desse padrão. Essa associação foi mais forte na análise múltipla após ajuste para idade, sexo e estado nutricional, tanto para os TG $(\mathrm{OR}=1,88$; IC95\%=1,10-3,22, p=0,021) quanto para o HDL-c (OR: 1,62; IC95\%: 1,01-2,62, p=0,047). Não foram encontradas associações estatisticamente significativas entre os Padrões Não Saudável e Tradicional com o perfil lipídico (dados não mostrados).

Tabela 1. Características demográficas, econômica, estado nutricional e perfil lipídico de adolescentes de escolas públicas, Salvador, Bahia, Brasil, 2009.

\begin{tabular}{lll}
\hline Variáveis & $\mathbf{n}$ & $\mathbf{\%}$ \\
\hline Sexo & & \\
Masculino & 479 & 40,3 \\
Feminino & 709 & 59,7 \\
Idade & & \\
$\geq 14$ anos & 647 & 54,5 \\
$<14$ anos & 541 & 45,5 \\
Categoria econômica & & \\
Classes C e D & 572 & 48,6 \\
Classe E & 577 & 49,8 \\
Estado Nutricional & & \\
Magreza & 89 & 7,5 \\
Eutrofia & 919 & 77,4 \\
Sobrepeso & 110 & 9,3
\end{tabular}


Research, Society and Development, v. 10, n. 14, e346101421840, 2021

(CC BY 4.0) | ISSN 2525-3409 | DOI: http://dx.doi.org/10.33448/rsd-v10i14.21840

Obesidade

CT

Normal

Limítrofe

Alto

LDL-c

Normal

Limítrofe

Alto

HDL-c

Normal

Limítrofe

Baixo

TG

Normal

953

150

85
5,9

80,1

14,0

5,9

58,8

35,4

5,8

13,9

60,4

25,7

80,2

12,6

Alto $85 \quad 7,2$

LDL-c: Lipoproteína de Baixa Densidade; HDL-c: Lipoproteína de Alta Densidade; CT: Colesterol Total; TG: Triglicerídeos. Fonte: Pesquisa Direta.

Tabela 2. Cargas fatoriais, comunalidade e variâncias dos três principais padrões alimentares praticados por adolescentes de escolas públicas, Salvador, Bahia, Brasil, 2009.

\begin{tabular}{lllll}
\hline Alimento/Grupo Alimentar & $\begin{array}{l}\text { Padrão } \\
\text { Saudável }\end{array}$ & $\begin{array}{l}\text { Não } \\
\text { Saudável }\end{array}$ & $\begin{array}{l}\text { Padrão } \\
\text { Tradicional }\end{array}$ & Comunalidade \\
\hline Lanches & $\mathbf{0 , 7 9 6}$ & 0,316 & 0,158 & 0,758 \\
Açúcar/Doces & $\mathbf{0 , 7 5 9}$ & 0,277 & 0,170 & 0,683 \\
Produtos Lácteos & $\mathbf{0 , 7 3 9}$ & 0,215 & 0,084 & 0,600 \\
Bebidas Artificiais & $\mathbf{0 , 7 1 8}$ & 0,175 & 0,106 & 0,557 \\
Comidas Típicas & $\mathbf{0 , 6 6 7}$ & 0,251 & 0,013 & 0,509 \\
Molhos & $\mathbf{0 , 6 3 9}$ & 0,144 & 0,277 & 0,506 \\
Bolos/Biscoitos & $\mathbf{0 , 6 0 3}$ & 0,355 & 0,234 & 0,544 \\
Óleos/Gorduras & $\mathbf{0 , 5 5 0}$ & 0,267 & 0,328 & 0,481 \\
Verduras/Legumes & 0,145 & $\mathbf{0 , 7 1 4}$ & 0,223 & 0,581 \\
Frutas & 0,435 & $\mathbf{0 , 6 4 3}$ & 0,090 & 0,611 \\
Raízes/Tubérculos & 0,361 & $\mathbf{0 , 6 1 0}$ & $-0,016$ & 0,502 \\
Peixes & 0,208 & $\mathbf{0 , 5 5 3}$ & 0,113 & 0,362 \\
Carne Vermelha & 0,418 & $\mathbf{0 , 4 7 9}$ & 0,345 & 0,523
\end{tabular}




$\begin{array}{lllll}\text { Pão } & 0,077 & -0,007 & \mathbf{0 , 7 3 9} & 0,552 \\ \text { Feijão } & 0,108 & 0,082 & \mathbf{0 , 6 8 1} & 0,482 \\ \text { Cereais } & 0,299 & 0,359 & \mathbf{0 , 6 2 3} & 0,606 \\ \text { Café/Chá } & -0,012 & 0,303 & \mathbf{0 , 5 7 8} & 0,426 \\ \text { Leite } & 0,421 & -0,035 & \mathbf{0 , 5 5 0} & 0,481 \\ \text { Frango/Ovos } & 0,377 & 0,393 & \mathbf{0 , 4 4 5} & 0,495 \\ \text { Variância explicada (\%) } & 39,90 & 8,72 & 5,35 & \\ \text { Variância acumulada (\%) } & 39,90 & 48,62 & 53,98 & \end{array}$

Valores em negrito correspondem às cargas fatoriais de cada alimento ou grupo alimentar no seu respectivo padrão. Fonte: Pesquisa Direta.

Tabela 3. Regressões politômicas brutas (simples) e ajustadas (múltiplas) entre Padrão Saudável e níveis lipídicos em adolescentes de escolas públicas, Salvador, Bahia, Brasil, 2009.

\begin{tabular}{|c|c|c|c|c|c|}
\hline \multicolumn{6}{|c|}{$\begin{array}{c}\text { CT (mg/dL) } \\
\text { ODDS RATIO (OR) / p-valor } \\
\text { Intervalo de Confiança (IC) } 95 \%\end{array}$} \\
\hline \multirow[b]{2}{*}{ Padrão Saudável } & \multirow{2}{*}{$\begin{array}{c}\text { Normal } \\
\text { Ref. }\end{array}$} & \multicolumn{2}{|c|}{ Limítrofe } & \multicolumn{2}{|c|}{ Alto } \\
\hline & & Bruta & Ajustada $^{\#}$ & Bruta & Ajustada $^{\#}$ \\
\hline $1^{\circ}$ tercil & & $\begin{array}{c}0,90 \quad 0,622 \\
(0,59-1,36)\end{array}$ & $\begin{array}{l}0,89 \quad 0,597 \\
(0,58-1,36)\end{array}$ & $\begin{array}{c}1,68 \quad 0,090 \\
(0,92-3,08)\end{array}$ & $\begin{array}{c}1,68 \quad 0,094 \\
(0,91-3,10)\end{array}$ \\
\hline $2^{\circ}$ tercil & & $\begin{array}{ll}1,23 & 0,294 \\
(0,83-1,83)\end{array}$ & $\begin{array}{l}1,20 \quad 0,364 \\
(0,80-1,78)\end{array}$ & $\begin{array}{ll}1,27 & 0,461 \\
(0,66-2,42)\end{array}$ & $\begin{array}{cc}1,28 & 0,460 \\
(0,66-2,46)\end{array}$ \\
\hline $3^{\circ}$ tercil & Ref. & Ref. & Ref. & Ref. & Ref. \\
\hline $\begin{array}{l}\text { Akaike Information Criterion } \\
\text { (AIC) }\end{array}$ & & 1477,701 & 1461,528 & 1477,701 & 1461,528 \\
\hline & & $\begin{array}{r}\text { LDL-c } \\
\text { ODDS RATI } \\
\text { Intervalo de Co }\end{array}$ & $\begin{array}{l}\text { /dL) } \\
\text { R) / p-valor } \\
\text { nça (IC) } 95 \%\end{array}$ & & \\
\hline & Normal & & ofe & & \\
\hline & Ref. & Bruta & Ajustada $^{\#}$ & Bruta & Ajustada $^{\#}$ \\
\hline $1^{\circ}$ tercil & & $\begin{array}{cc}1,11 & 0,472 \\
(0,82-1,50)\end{array}$ & $\begin{array}{cc}1,12 & 0,457 \\
(0,83-1,51)\end{array}$ & $\begin{array}{cc}1,38 & 0,307 \\
(0,74-2,58)\end{array}$ & $\begin{array}{c}1,38 \quad 0,313 \\
(0,73-2,58)\end{array}$ \\
\hline $2^{\circ}$ tercil & & $\begin{array}{cc}1,26 & 0,115 \\
(0,94-1,70)\end{array}$ & $\begin{array}{cc}1,28 & 0,104 \\
(0,95-1,73)\end{array}$ & $\begin{array}{c}1,45 \quad 0,236 \\
(0,78-2,72)\end{array}$ & $\begin{array}{c}1,43 \quad 0,262 \\
(0,76-2,70)\end{array}$ \\
\hline $3^{\circ}$ tercil & Ref. & Ref. & Ref. & Ref. & Ref. \\
\hline $\begin{array}{l}\text { Akaike Information Criterion } \\
\text { (AIC) }\end{array}$ & & 2016,005 & 2006,373 & 2016,005 & 2006,373 \\
\hline
\end{tabular}

HDL-c $(\mathbf{m g} / \mathbf{d L})$ 


\begin{tabular}{|c|c|c|c|c|c|}
\hline \multirow[b]{3}{*}{$1^{\circ}$ tercil } & \multirow{3}{*}{$\begin{array}{c}\text { Normal } \\
\text { Ref. }\end{array}$} & \multicolumn{2}{|c|}{ Limítrofe } & \multicolumn{2}{|c|}{ Baixo } \\
\hline & & Bruta & Ajustada $^{\#}$ & Bruta & Ajustada $^{\#}$ \\
\hline & & $(0,71-1,64)$ & $(0,74-1,73)$ & $(0,93-2,37)$ & $(1,01-2,62)$ \\
\hline $2^{\circ}$ tercil & & $\begin{array}{c}1,01 \quad 0,949 \\
(0,67-1,52)\end{array}$ & $\begin{array}{c}1,05 \quad 0,782 \\
(0,70-1,59)\end{array}$ & $\begin{array}{cc}1,05 & 0,835 \\
(0,66-1,67)\end{array}$ & $\begin{array}{c}1,11 \quad 0,650 \\
(0,69-1,79)\end{array}$ \\
\hline $3^{\circ}$ tercil & Ref. & Ref. & Ref. & Ref. & Ref. \\
\hline $\begin{array}{l}\text { Akaike Information Criterion } \\
\text { (AIC) }\end{array}$ & & 2210,717 & 2155,799 & 2210,717 & 2155,799 \\
\hline \multicolumn{6}{|c|}{$\begin{array}{c}\text { TG (mg/dL) } \\
\text { ODDS RATIO (OR) / p-valor } \\
\text { Intervalo de Confiança (IC) } 95 \%\end{array}$} \\
\hline & Normal & \multicolumn{2}{|c|}{ Limítrofe } & \multicolumn{2}{|c|}{ Alto } \\
\hline & \multirow[t]{2}{*}{ Ref. } & Bruta & Ajustada $^{\#}$ & Bruta & Ajustada $^{\#}$ \\
\hline $1^{\circ}$ tercil & & $\begin{array}{c}1,41 \quad 0,126 \\
(0,90-2,19)\end{array}$ & $\begin{array}{ll}1,41 & 0,123 \\
(0,90-2,20)\end{array}$ & $\begin{array}{c}1,80 \quad 0,028 \\
(1,06-3,07)\end{array}$ & $\begin{array}{cc}1,88 & 0,021 \\
(1,10-3,22)\end{array}$ \\
\hline $2^{\circ}$ tercil & & $\begin{array}{c}1,51 \quad 0,060 \\
(0,98-2,33)\end{array}$ & $\begin{array}{c}1,48 \quad 0,074 \\
(0,96-2,29)\end{array}$ & $\begin{array}{c}0,91 \quad 0,770 \\
(0,49-1,67)\end{array}$ & $\begin{array}{c}0,91 \quad 0,773 \\
(0,49-1,67)\end{array}$ \\
\hline $3^{\circ}$ tercil & Ref. & Ref. & Ref. & Ref. & Ref. \\
\hline $\begin{array}{l}\text { Akaike Information Criterion } \\
\text { (AIC) }\end{array}$ & & 1489,731 & 1469,172 & 1489,731 & 1469,172 \\
\hline
\end{tabular}

\# Ajustado por idade, sexo e estado nutricional. Fonte: Pesquisa Direta.

\section{Discussão}

O presente estudo identificou e caracterizou três padrões alimentares, que representam a variabilidade de 53,98\% do consumo alimentar de adolescentes de escolas públicas de Salvador-Bahia. Esta variabilidade expressa a participação de 39,90\% do Padrão Não Saudável, 8,72\% do Padrão Saudável e 5,35\% do Padrão Tradicional indicando que estes padrões expressam com $95 \%$ de segurança a realidade encontrada na população. Este resultado foi similar ao estudo de Ribeiro-Silva et al., (2017) e de Brito et al., (2020) e sendo maior que outras pesquisas realizadas com adolescentes no Brasil (Vaz et al., 2018; Borges et al., 2015) e em outros países (Perng et al., 2017; Kelishadi et al., 2018; Khayatzadeh et al., 2018; Zhen et al., 2018). Vale ressaltar que a comparação dos nossos resultados com outros estudos deve ser realizada com cautela, em decorrência dos inúmeros métodos empregados para extração dos padrões e pelas diferenças nas características das populações.

O Padrão Saudável foi composto por altas cargas fatoriais de frutas, legumes, verduras, raízes e tubérculos e carga fatorial baixa para carne vermelha, semelhante ao padrão identificado no estudo de Vaz et al. (2018), realizado com adolescentes brasileiros aos 18 anos. No presente estudo, a menor adesão ao Padrão Saudável associou-se com menores níveis de HDL-c e maiores de TG, diferentemente dos resultados de Vaz et al., (2018), que não encontraram associação entre o padrão saudável e variáveis lipídicas.

Concordando com o presente trabalho, estudos de revisão sistemática encontraram que os padrões alimentares "Saudáveis", identificados nos estudos avaliados, foram compostos principalmente por legumes, verduras, frutas, peixes, carboidratos complexos e fibras dietéticas, além de nutrientes antioxidantes (Cunha et al., 2018). O consumo desses alimentos traz benefícios para a saúde, devido a presença de nutrientes antioxidantes, polifenóis, fibras e outras características, as quais 
contribuem para redução da oxidação lipídica nas artérias, menor resistência vascular, além de melhorar a função endotelial e, consequentemente, reduzir o risco de Doenças Cardiovasculares (DCV) e suas complicações (Miller et al., 2017).

Estudos relatam que o consumo de alimentos com maior densidade energética, alto conteúdo de gorduras e açúcares com elevado índice glicêmico, característicos do Padrão Não Saudável, está diretamente associado ao rápido aumento de glicose pós-prandial. Essa situação leva à liberação substancial de insulina e aumento da lipogênese, aumentando a captação de glicose pelas células adiposas via recrutamento de transportadores de glicose para a membrana plasmática assim como ativando enzimas glicolíticas e lipogênicas. Presume-se que quanto mais alta a glicemia pós-prandial, maior probabilidade de aumento na síntese de ácidos graxos a partir da glicose, devido ao fornecimento excessivo de átomos de carbono para o fígado (Polacow \& Junior, 2007). Em consequência disso pode ocorrer oxidação reduzida e ao maior acúmulo de ácidos graxos nos tecidos e no sangue e a secreção de lipoproteínas de muito baixa densidade (VLDL - Very Low Density Lipoprotein), podendo contribuir para aumento nos níveis de TG e LDL-c e diminuição das concentrações de HDL-c (Alves et al., 2019; Brito et al., 2020; Beserra, et al., 2020). No entanto, no presente estudo não foi encontrada associação entre Padrão não Saudável e variáveis lipídicas.

O Padrão Tradicional identificado no presente estudo é caracterizado por alimentos presentes na alimentação dos brasileiros, semelhante àqueles padrões identificados em outras investigações realizadas com adolescentes (Dishchekenian et al., 2011; Vaz et al., 2018; Mascarenhas et al., 2016). Alves et al. (2019) e Borges et al. (2015) também encontraram padrão "Tradicional" com itens similares ao do presente estudo, destacando-se o arroz e feijão, como alimentos mais consumidos pelos adolescentes brasileiros. Apesar deste estudo não ter encontrado associação entre o Padrão Tradicional e alterações lipídicas, torna-se importante relatar que alguns alimentos que o compõem, como o pão branco, arroz branco, macarrão e farinha de mandioca, podem impactar de forma negativa os níveis lipídicos, pois devido ao grau de refinamento, estes alimentos são metabolizados rapidamente em carboidratos simples, provocando maior elevação da concentração de glicose e insulina no sangue, causando um quadro metabólico adverso de níveis de HDL-c baixo e de TG altos (Kelishadi et al., 2018).

Considerando a população em estudo, torna-se importante destacar que o perfil lipídico dos adolescentes é influenciado por características peculiares a essa faixa etária, como alterações hormonais durante a puberdade e maturação sexual, o que pode alterar resultados de associações entre dieta e fatores de risco cardiometabólicos (Carvalho et al., 2016). A literatura relata que a concentração das variáveis do perfil lipídico muda de acordo o estágio de maturação sexual do adolescente e os diferentes pontos de corte para avaliação geralmente não consideram os estágios da puberdade. Por isso, mesmo havendo referências brasileiras atualizadas para avaliação do perfil lipídico de adolescentes, no presente estudo optamos por utilizar os pontos de corte de Jolliffe e Janssen (2006) que consideram as flutuações naturais nas concentrações de lipoproteínas durante a puberdade.

A prevalência de excesso de peso e alterações no perfil lipídico identificado no presente estudo é relevante. Vale destacar que hábitos alimentares inadequados contribuem para o excesso de peso e alteração no perfil lipídico os quais constituem fatores de risco para o desencadeamento de DCV (Carvalho et al., 2016; Zhang et al., 2015). As práticas alimentares possuem repercussões importantes na saúde e no estado nutricional do indivíduo, sendo necessários estudos que avaliem esta associação e apresentem resultados que promovam mudanças na escolha e no preparo dos alimentos de maneira que seja adotada uma alimentação mais saudável.

Assim, a educação nutricional na adolescência deve ressaltar a importância do papel da alimentação saudável sobre a qualidade de vida e, acima de tudo, possibilitar a autonomia nas escolhas alimentares para que os benefícios sejam alcançados em longo prazo. Ações integradas devem ser realizadas, envolvendo a participação da família, escola, comunidade e a mídia para facilitar a promoção de saúde, priorizando a prevenção de doenças e a adoção de um estilo de vida saudável (Pereira, Pereira \& Angelis-Pereira, 2017). 
Entre as limitações do presente estudo destaca-se que padrões alimentares variam de acordo com sexo, idade, cultura, condições socioeconômicas e disponibilidade alimentar, o que torna subjetiva a comparação com outras populações. Além disso, distintos métodos são utilizados para avaliar padrão alimentar nos estudos, dificultando a comparação entre os resultados. Os pontos fortes de nosso estudo são a utilização do QFA validado e publicado além da população amostral probabilística. Vale destacar também o tratamento estatístico robusto, tanto na obtenção dos padrões quanto na avaliação das associações, com utilização da regressão logística politômica que permitiu avaliar a variável resposta de forma categorizada, possibilitando avaliação minuciosa dos resultados obtidos sobre a relação exposição-doença.

Apesar da existência da lacuna temporal entre o período da coleta dos dados e os resultados apresentados neste artigo possam ser aparentemente uma limitação é reconhecido que o propósito de uso de algumas informações em saúde e a temporalidade deixa de ser questão impeditiva para consideração dos resultados de análise de muitas informações em arquivos depositados em unidades acadêmicas. Assim, sobressai o conhecimento sobre o consumo dietético e perfil lipídico, particularmente com o risco cardiovascular, relação que teve seus estudos iniciados com as pesquisas de Framingham nos anos 40 envolvendo grupos familiares (Dawber, Meadors \& Moore, 1951). Embora este conhecimento seja universal e relações são estabelecidas para a população adulta, muitas lacunas são identificadas para grupos populacionais específicos a exemplo dos adolescentes. Neste contexto, informação similar é difícil de se tornar obsoleta em especial quando não se trata de avaliar a prevalência do evento com escassa investigação sobre o tema e quando são adotadas técnicas estatísticas robustas para análise da associação investigada.

\section{Conclusão}

Foi identificado associação entre menor aderência ao Padrão Saudável e baixa concentração sérica de Lipoproteína de Alta Densidade e elevada concentração sérica de triglicerídeos. Estudar padrões alimentares associado com desfechos na saúde torna-se necessário na infância e adolescência por ser uma fase da vida tão particular em suas características, sendo necessário que práticas alimentares inadequadas sejam desencorajadas e medidas preventivas sejam planejadas e executadas buscando reduzir o risco cardiometabólico na vida adulta.

\section{Referências}

Akaike, H. (1971). Information theory and an extension of the maximum likelihood principle. In: Petrov BN, Csaki F. (Ed). Proceedings of the second international symposium on information theory. (p. 199-213). Armenia.

Alcântara Neto, O. D., Silva, R. C. R., Assis, A. M., Pinto, J. (2012). Factors associated with dyslipidemia in children and adolescents enrolled in public schools of Salvador, Bahia. Rev Bras Epidemiol,15(2), 335-45. Doi: 10.1590/S1415-790X2012000200011.

Alves, M. A., Souza, A. M., Barufaldi, L. A., Tavares, B. M., Bloch, K. V., Vasconcelos, F. A. (2019). Dietary patterns of Brazilian adolescents according to geographic region: an analysis of the Study of Cardiovascular Risk in Adolescents (ERICA). Cad Saúde Pública, 35(6): e00153818. Doi: 10.1590/0102$311 \mathrm{X} 00153818$

Associação Brasileira de Empresas de Pesquisa (2008). Critério de Classificação Econômica Brasil (CCEB). Acessado em agosto 31 , 2019. http://www.abep.org/criterio-brasil.

Berenson, G. S., Srinivasan, S. R., Xu, J. H., Chen, W. (2016). Adiposity and cardiovascular risk factor variables in childhood are associated with premature death from coronary heart disease in adults: the Bogalusa Heart Study. Am J Med Sci, 352(5), p. 448-454. Doi: 10.1016/j.amjms.2016.08.006.

Beserra, J. B., Soares, N. I. S., Marreiros, C. S., Carvalho, C. M. R. G., Martins, M. C. C. ...Frota, K. M G. (2020). Crianças e adolescentes que consomem alimentos ultraprocessados possuem pior perfil lipídico? Uma revisão sistemática. Ciência \& Saúde Coletiva, 25(12), 4979-499. Doi:10.1590/1413812320202512.29542018 .

Borges, C. A., Rinaldi, A. E., Conde, W. L., Mainardi, G. M., Behar, D., Slater, B. (2015). Dietary patterns: a literature review of the methodological characteristics of the main step of the multivariate analyzes. Rev Bras Epidemiol, 18(4), 837-57. Doi: 10.1590/1980-5497201500040013.

Brito, A. N. M., Conde, W. L., Frota, K. M. G., Silva, A. R. V., Lima, L. H. O. (2020). Padrão alimentar e resistência à insulina em adolescentes. Research,Society and Development, 9(12). Doi: 10.33448/rsd-v9i12.10786. 
Carvalho, C. A., Fonsêca, P. C., Nobre, L. N., Priore, S. E., Franceschini, C. (2016). Methods of a posteriori identification of food patterns in Brazilian children: a systematic review. Cienc Saude Colet, 21(1), 143-54. Doi: 10.1590/1413-81232015211.18962014.

Corrêa, R. S., Vencato, P. H., Rockett, F. C., Bosa, V. L. (2017). Dietary patterns: are there differences between children and adolescents? Cienc Saude Colet, 22(2), 553-562. Doi: 10.1590/1413-81232017222.09422016.

Cunha, C. M., Costa, P. R., de Oliveira, L. P., Queiroz, V. A., Pitangueira, J. C., Oliveira, A. M. (2018). Dietary patterns and cardiometabolic risk factors among adolescents: systematic review and meta-analysis. Br J Nutr, 119(8), 859-79. Doi: 10.1017/S0007114518000533.

Cunha, D. B., Bezerra, I. N., Pereira, R. A., Sichieri, R. (2018). At-home and away-from-home dietary patterns and BMI z-scores in Brazilian adolescents. Appetite, 120, 374-80. Doi: 10.1016/j.appet.2017.09.028

Dawber, T. R., Meadors, G. F., Moore, F. E. (1951). Epidemiological Approaches to Heart Disease: The Framingham Study. Am J Public Health, $41,279-286$. Doi: $10.2105 \%$ Fajph.41.3.279

Dishchekenian, V. R., Escrivão, M. A., Palma, D., Ancona-Lopez, F., de Araújo, E. A., Taddei, J. A. (2011). Dietary patterns of obese adolescents and different metabolic effects. Rev Nutr,24(1), 17-29. Doi: 10.1590/S1415-52732011000100002.

Enes, C. C., Silva, J. R. (2018). Association between excess weight and serum lipid alterations in adolescents. Cienc Saude Colet, 23(12), 4055-63. Doi: $10.1590 / 1413-812320182312.27882016$.

Faludi, A. A., Izar, M. C., Saraiva, J. F., Chacra, A. P., Bianco, H. T., Afiune, A. ... Salgado, F. S. (2017). Atualização da Diretriz Brasileira de Dislipidemias e Prevenção da Aterosclerose. Arq Bras Cardiol, 109(2) Supl 1), 1-76. Disponível em: http://publicacoes.cardiol.br

Greenland, S. (2008). Introduction to Regression Modeling. In: Rothman KJ, Greenland S, Lash TL. (p. 424-427). Philadelphia: Modern epidemiology.

Hair, J. F., Black, W. C., Babin, B. J., Anderson, R. E., Taham, R. L. (2009). Análise multivariada de dados. In: Análise Fatorial (p. 103-124). Porto Alegre: Bookman Companhia.

Jolliffe, C. J., Janssen, I. (2006). Distribution of lipoproteins by age and gender in adolescents. Circulation, 114(10), 1056-62. Doi: 10.1161/CIRCULATIONAHA.106.620864

Kelishadi, R., Heshmat, R., Mansourian, M., Motlagh, M. E., Ziaodini, H., Taheri, M. ... Mozafarian, N. (2018). Association of dietary patterns with continuous metabolic syndrome in children and adolescents; a nationwide propensity score-matched analysis: the CASPIAN-V study. Diabetol Metab Syndr, 10, 52. Doi: 10.1186/s13098-018-0352-3.

Khayyatzadeh, S. S., Bagherniya, M., Fazeli, M., Khorasanchi, Z., Bidokhti, M. S., Ahmadinejad, M. ... Ghayour-Mobarhan, M. (2018). A Western dietary pattern is associated with elevated level of high sensitive C-reactive protein among adolescent girls. Eur J Clin Invest, 48(4):e12897. Doi: 10.1111/eci.12897

Lartey, A., Marquis, G. S., Aryeetey, R., Nti, H. (2018). Lipid profile and dyslipidemia among school-age children in urban Ghana. BMC Public Health, 18(1), 320. Doi: 10.1186/s12889-018-5196-0.

Lohman, T. G., Roche, A. F., Martorell, R. (Ed.) (1988). Anthropometric standardization reference manual. Abridged edition. Champaign: Human Kinetics Publishers.

Magnussen, C. G., Raitakari, O. T., Thomson, R., Juonala, M., Patel, D. A., Viikari, J. S. ... Venn, A. (2008). Utility of currently recommended pediatric dyslipidemia classifications in predicting dyslipidemia in adulthood: evidence from the Childhood Determinants of Adult Health (CDAH) study, Cardiovascular Risk in Young Finns Study, and Bogalusa Heart Study. Circulation, 117(1), 32-42. Doi: 10.1161/CIRCULATIONAHA.107.718981

Mascarenhas, J. M., Silva, R. C., Machado, M. E., Santos, C. A., Marchioni, D. M., Barreto, M. L. (2016). Validation of a food frequency questionnaire designed for adolescents in Salvador, Bahia, Brazil. Rev Nutr, 29(2), 163-71. Doi: 10.1590/1678-98652016000200002.

Miller, V., Mente, A., Dehghan, M., Rangarajan, S., Zhang, X., Swaminathan, S. .. Yusuf S. (2017). Prospective Urban Rural Epidemiology (PURE) study investigators. Fruit, vegetable, and legume intake, and cardiovascular disease and deaths in 18 countries (PURE): a prospective cohort study. Lancet, 390(10107), 2037-49. Doi: 10.1016/S0140-6736(17)32253-5.

Mirmiran, P., Ziadlou, M., Karimi, S., Hosseini-Esfahani, F., Azizi, F. (2019). The association of dietary patterns and adherence to WHO healthy diet with metabolic syndrome in children and adolescents: Tehran lipid and glucose study. BMC Public Health, 19(1), 1457. Doi: 10.1186/s12889-019-7779-9.

Neumann, A. I., Martins, I. S., Marcopito, L. F., Araujo, E. A. (2007). Dietary patterns associated with risk factors for cardiovascular disease in a Brazilian city. Rev Panam Salud Publica, 22(5), 329-39. Doi: 10.1590/s1020-49892007001000006.

Pereira, T. S., Pereira, R. C., Angelis-Pereira, M. C. (2017). Influência de intervenções educativas no conhecimento sobre alimentação e nutrição de adolescentes de uma escola pública. Cienc Saude Colet,22(2),427-435. https://doi.org/10.1590/1413-81232017222.16582015.

Perng, W., Fernandez, C., Peterson, K.E., Zhang, Z., Cantoral, A., Sanchez, B.N., Solano-González, M., Téllez-Rojo, M. M., Baylin, A. (2017). Dietary patterns exhibit sex-specific associations with adiposity and metabolic risk in a cross-sectional study in urban Mexican adolescents. $J$ Nutr, 147(10), 1977-85. Doi:10.3945/jn. 117.256669.

Polacow, V. O., Junior, A. H. L. (2007). Dietas hiperglicídicas: Efeitos da substituição isoenergética de gordura por carboidratos sobre o metabolism de lipídios, adiposidade corporal e sua associação com atividade física e com o risco de Doença Cardiovascular. Arq Bras Endocrinol Metab, 51(3), 389-399. Doi: 10.1590/S0004-27302007000300006.

Ribeiro-Silva, R. C., Fiaccone, R. L., Conceição-Machado, M. E., Ruiz, A. S., Barreto, M. L., Santana, M. L. (2018). Body image dissatisfaction and dietary patterns according to nutritional status in adolescents. J Pediatr, 94(2), 155-61. Doi: 10.1016/j.jped.2017.05.005. 
Research, Society and Development, v. 10, n. 14, e346101421840, 2021

(CC BY 4.0) | ISSN 2525-3409 | DOI: http://dx.doi.org/10.33448/rsd-v10i14.21840

Vaz, J. D., Buffarini, R., Kac, G., Bielemann, R.M., Oliveira, I., Menezes, A.B., Assunção, M. C. F. (2018). Dietary patterns are associated with blood lipids at 18-year-olds: a cross-sectional analysis nested in the 1993 Pelotas (Brazil) birth cohort. Nutr J, 17(1), 77. Doi: 10.1186/s12937-018-0389-z.

White, D. A., Anand, G. M., Qayum, O., Ibezim, C. F., Sherman, A. K., Raghuveer, G. (2019). Modifiable clinical correlates of vascular health in children and adolescents with dyslipidemia. Pediatr Cardiol, 40(4), 805-12. Doi: 10.1007/s00246-019-02071-w.

World Health Organization (2007). Growth reference data for 5-19 years. Geneva: World Health Organization. Disponível em: https://www.who.int/tools/growth-reference-data-for-5to19-years.

Zhang, Z., Gillespie, C., Welsh, J. A., Hu, F. B., Yang, Q. (2015). Usual intake of added sugars and lipid profiles among the U.S. adolescents: National Health and Nutrition Examination Survey, 2005 2010. J Adolesc Health, 56(3), 352-9. Doi: 10.1016\%2Fj.jadohealth.2014.12.001.

Zhen, S., Ma, Y., Zhao, Z., Yang, X., Wen, D. (2018). Dietary pattern is associated with obesity in Chinese children and adolescents: data from China Health and Nutrition Survey (CHNS). Nutr J, 17(1), 68. Doi: 10.1186/s12937-018-0372-8 